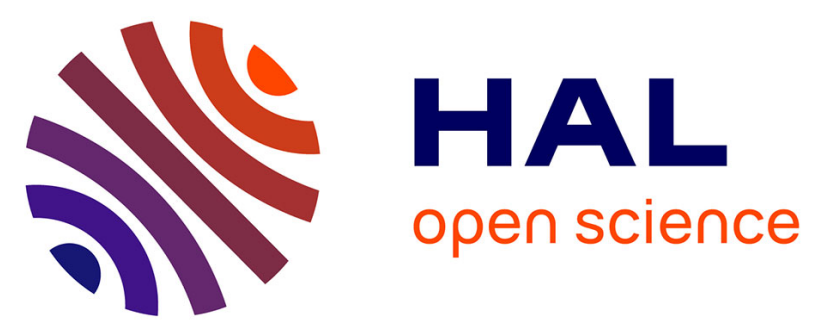

\title{
Reliability Analysis of Mixed Mode Cracking with Viscoelastic Orthotropic Behaviour
}

\author{
Rostand Moutou Pitti, Alaa Chateauneuf, Claude Chazal
}

\section{To cite this version:}

Rostand Moutou Pitti, Alaa Chateauneuf, Claude Chazal. Reliability Analysis of Mixed Mode Cracking with Viscoelastic Orthotropic Behaviour. Tom Proulx. Mechanics of Time-Dependent Materials and Processes in Conventional and Multifunctional Materials, Proceedings of the 2011 Annual Conference on Experimental and Applied Mechanics, 3, Springer, pp.249-256, 2011, Conference Proceedings of the Society for Experimental Mechanics Series, 978-1-4614-0213-8. 10.1007/978-1-4614-0213-8_36 . hal-01616927

\section{HAL Id: hal-01616927 https://hal.science/hal-01616927}

Submitted on 12 Jan 2018

HAL is a multi-disciplinary open access archive for the deposit and dissemination of scientific research documents, whether they are published or not. The documents may come from teaching and research institutions in France or abroad, or from public or private research centers.
L'archive ouverte pluridisciplinaire HAL, est destinée au dépôt et à la diffusion de documents scientifiques de niveau recherche, publiés ou non, émanant des établissements d'enseignement et de recherche français ou étrangers, des laboratoires publics ou privés. 


\title{
RELIABILITY ANALYSIS OF MIXED MODE CRACKING WITH VISCOELASTIC ORTHOTROPIC BEHAVIOUR
}

\author{
Rostand MOUTOU PITTI ${ }^{\mathrm{a}}$, Alaa CHATEAUNEUF ${ }^{\mathrm{a}}$, Claude CHAZAL ${ }^{\mathrm{b}}$. \\ ${ }^{a}$ Clermont Université, Université Blaise Pascal, Laboratoire de Mécanique et Ingénieries EA \\ 3867, BP 206, 63000 Clermont Ferrand, France. \\ ${ }^{\mathrm{b}}$ GEMH Laboratory, Limoges University, \\ Centre Universitaire de Génie Civil, 19300 Egletons, France. \\ Rostand.moutou.pitti@polytech.univ-bpclermont.fr
}

\begin{abstract}
The reliability analysis applied to viscoelastic and orthotropic materials, in the case of mixed mode configuration, is studied in this work. The $\mathrm{M}$ integral, separating mixed mode during creep crack initiation in viscoelastic field, is used in the analytical approach. The main development, based on conservative law, and a combination of real and virtual displacement fields, is proposed. In order to provide mixed mode configuration, a Compact Tension Shear (CTS) specimen is used in the numerical process. Simultaneously the fracture and the viscoelastic procedures are coupled with reliability analysis in order to take account for model and parameter uncertainties. In this case, the random parameters related to model factors, elastic constants are defined in the reliability analysis of time dependent fracture materials subjected to complex loading. As results, the reliability levels are computed and discussed according to various mixed-mode loading scenarios
\end{abstract}

\section{Introduction}

The mixed mode conditions often result from bending loads that are imposed on the structural component, and they are generally aggravated by the heterogeneous and orthotropic character, and the viscoelastic behavior of the material [1]. In the several cases, the negligence of mixed mode interaction in the design of composite structures may lead to significant errors in strength predictions, and mixed mode fracture criteria are thus of great importance for predicting failure of notched wood components. However, the mixed modes cracks, combining with the time depend behavior, are phenomena affected by high uncertainties, where deterministic methods fail to predict accurately the structural life.

The objective of the current investigation is to apply the reliability model to the behavior of viscoelastic orthotropic material in order to estimate the uncertainties of the used fracture parameters.

In viscoelastic approach, path independent integrals have been used in order to study the impact of the mixed mode ratio in crack initiation [2] and crack growth process in wooden material [3]. In the literature, several authors have applied the reliability theory to fracture mechanics problems [4]. In the past, the reliability approach has been coupled with the boundary element model for probabilistic fatigue life assessment in crack propagation mode mixty [5] and recently, random fatigue crack growth in mixed mode has been studied by stochastic collocation method [6]. However, these works don't take into account the viscoelastic effects.

In the first part of the paper, the reliability approach is recalled. In this case, the failure probability function and the First Order Reliability Method (FORM) are defined. In the second part, the conservative laws [7], combining the real and virtual mechanical fields [8] and the non-dependant integral parameter $M \theta$ in crack initiation process, is recalled. The time dependent effects are introduced by the generalized Kelvin Voigt model resolving by an incremental viscoelastic formulation [2]. After, the CTS (Compact Tension Shear) specimen [9, 10] allows the mixed mode configuration is described. The subroutine of the crack initiation and reliability process is based on an energetic criterion. The random parameters used in the numerical model are fixed according to the critical values of energy release rate in opening and shear mode. Finally, the reliability analysis provides us to obtained the failure probability and the sensitivity of the fracture parameters regarding these complex solicitation are posted. 


\section{Reliability analysis}

The reliability is defined as the capability of a structure to guarantee the purpose for which it has been designed, along the entire period of its lifetime $[11,12]$. The reliability is complementary to the failure probability $P_{f}$. The leading step in the reliability analysis consists in identifying the main set of random variables $X_{i}, i=1,2, \ldots, n$, for which uncertainties have to be considered. For all of these variables $X_{i}$, statistical distributions are defined in order to describe the uncertainties; a realization of this set of variables is noted $x_{i}$. The probabilistic distributions can be selected by physical observations, statistical studies, laboratory analysis and expert opinion. However, it is important to emphasize that the quality of the reliability assessment strongly depends on the quality of the available information (i.e. input data) as well as the quality of the reliability model.

The second step consists in defining the potentially critical failure modes. For each one of them, a limit state function $g\left(x_{i}\right)$ is defined to separate the behavior space into two regions: the safe domain, where $g\left(x_{i}\right)>0$, and the failure domain where $g\left(x_{i}\right)<0$. The boundary between these two domains, defined by $g\left(x_{i}\right)=0$, is known as the limit state itself. In our case, the analysis is carried out with respect to fatigue failure. Considering the fracture mechanics in mixed mode, the limit state can be written in the form:

$$
g\left(x_{i}\right)=1-\frac{{ }^{1} G_{v}\left(x_{i}\right)}{{ }^{1} G_{c}\left(x_{i}\right)}+\alpha \frac{{ }^{2} G_{v}\left(x_{i}\right)}{{ }^{2} G_{c}\left(x_{i}\right)}
$$

where ${ }^{1} G_{v},{ }^{1} G_{c},{ }^{2} G_{v}$ and ${ }^{2} G_{c}$ are respectively the applied and critical energy release rates for modes 1 and 2, and $\alpha$ is the orthotropic factor for the specimen, equal to one in our case. For this limit state, the probability of failure is evaluated by integrating the joint density function over the failure domain Erreur ! Source du renvoi introuvable.

$$
P_{f}=\operatorname{Pr} g\left[G\left(X_{i}\right) \leq 0\right]=\int_{g\left(x_{i}\right) \leq 0} f_{X_{1}, X_{2}, \ldots, X_{n}}\left(x_{1}, x_{2}, \ldots, x_{n}\right) d x_{1} d x_{2} \cdots d x_{n}
$$

where $f_{X_{1}, X_{2}, \ldots, X_{n}}\left(x_{1}, x_{2}, \ldots, x_{n}\right)$ is the joint density function of the variables $X_{i}$. The evaluation of the above integral is not possible in practical cases, because of the required high precision and the unavailability of the joint density function. For these reasons, two approaches are commonly proposed for mechanical reliability [13]: random simulations and approximation methods. The random simulations, known as Monte-Carlo methods [12], with or without variance reduction, require a large number of evaluations of the performance function, and consequently of the mechanical model. They are therefore convenient when the mechanical model is analytical, involving very low computation time. The precision of Monte-Carlo simulations can be controlled by evaluating the standard deviation of the probability estimate. When the finite element models are involved, the approximation methods, such as First Order Reliability Method FORM [12], become attractive as they require relatively low number of mechanical runs. However, the precision of FORM depends on the curvature of the performance function in the normalized Gaussian space, as this method is based on local approximation by hyper-plane in the neighborhood of the most probable failure point. Previous experience in mechanical engineering shows that the mechanical response is usually smooth and curvatures are low, especially in static analysis, which allows us to use FORM approximations.

In our case, the finite element model is used for mechanical analysis, and therefore the computation time for each evaluation of the performance function becomes significant. In this case, the use of Monte-Carlo simulations, requiring a very large number of mechanical analyses, leads to astronomic computation time, which is impossible in practical cases. To overcome this difficulty, we have applied the FORM algorithms as described in [12]. This procedure has been performed for the limit state function in Equation (1), where the energy release rates are computed by finite element analyses as described below.

\section{Integral parameter in viscoelastic material}


For uncoupling crack modes, some authors have developed an uncoupling fracture mode algorithm with a similar integral called M. This integral is also characterized by a non path dependence of the integration domain [8]. The main difference consists in combination of virtual $\mathrm{v}$ and real $\mathrm{u}$ singular fields according to the virtual work principle. Thus, they propose a bilinear form of the elastic strain energy as follow:

$$
F(u, v)=\frac{1}{2} \cdot \sigma_{i j}^{(u)} \cdot \varepsilon_{i j}^{(v)}=\frac{1}{2} \cdot \sigma_{i j}^{(v)} \cdot \varepsilon_{i j}^{(u)}
$$

$\sigma_{i j}^{(u)}, \sigma_{i j}^{(v)}, \varepsilon_{i j}^{(u)}, \varepsilon_{i j}^{(v)}$ are components of stress and strain tensors deduced from the real (induced u) and virtual (induced $\mathrm{v}$ given by Shi's singular forms) displacement fields, respectively. Without crack propagation and pressure on the crack lips, the M-integral form is given by :

$$
M=\frac{1}{2} \int_{\Gamma}\left(\sigma_{i j, 1}^{v} \cdot u_{i}-\sigma_{i j}^{u} \cdot v_{i, 1}\right) \cdot n_{j} \mathrm{~d} \Gamma
$$

The integral (4) is defined with a curvilinear integration domain. It's preferable, for a finite element implementation, to transform this form in terms of a surface integral by introducing a $\theta$ field [14], continuous and differentiable, ( $\theta_{1}=1$ and $\theta_{2}=0$ inside the ring and $\vec{\theta}=\overrightarrow{0}$ outside it), Figure 1 (b).

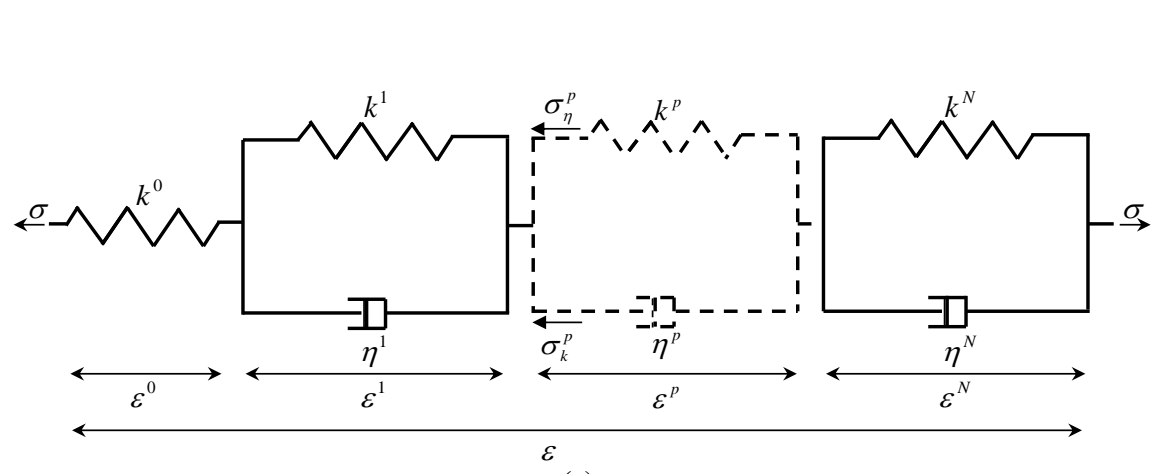

(a)

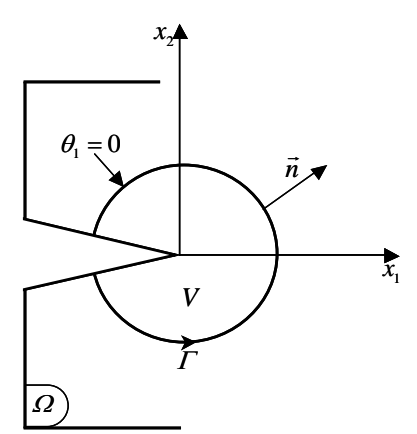

(b)

Figure 1 - (a): Generalized Kelvin Voigt model. (b): Integration domain

In these conditions, the relation (4) can be transformed by using the Gauss-Ostrogradski's transformation and balance equations. Hence, we obtain the M $\theta$ - integral defined as follow [14]

$$
M \theta=\frac{1}{2} \int_{V}\left(\sigma_{i j}^{u} \cdot v_{i, k}-\sigma_{i j, k}^{v} \cdot u_{i}\right) \cdot \theta_{k, j} \mathrm{~d} V
$$

The generalized Kelvin Voigt model is used in order to introduce viscoelastic properties, Figure 1 (a).

This rheological model is well adapted for time dependent representation because it allows uncoupling elastic properties (which induce free energy) and viscous properties (which induce energy dissipation). In this case, the expression (5) can be generalized for each elastic spring [2]:

$$
M \theta_{v}^{(p)}(u, v)=\frac{1}{2} \int_{V}\left(\sigma_{i j}^{(p)}(u) \cdot u_{i}^{(p)}-\sigma_{i j, k}^{(p)}(v) \cdot v_{i, k}^{(p)}\right) \cdot \theta_{k, j} \mathrm{~d} V \quad \text { with } p=(0,1, \ldots . M)
$$

In order to express the real stress intensity factors ${ }^{u} K_{I}^{(p)}$ and ${ }^{u} K_{I I}^{(p)}$, the complete uncoupling is obtained by performing two distinct calculations from the $M \theta_{v}^{(p)}(u, v)$-integral. With these considerations, judicious values for the virtual stress intensity factors ${ }^{v} K_{I}^{(p)}$ and ${ }^{v} K_{I I}^{(p)}$ have chosen as follow :

$$
{ }^{u} K_{I}^{(p)}=\frac{8 \cdot M \theta_{v}^{(p)}(u, v)\left({ }^{v} K_{I}^{(p)}=1,{ }^{v} K_{I I}^{(p)}=0\right)}{C_{1}^{(p)}} \quad \text { and } \quad{ }^{u} K_{I I}^{p}=\frac{8 \cdot M \theta_{v}^{(p)}(u, v)\left({ }^{v} K_{I}^{(p)}=0,{ }^{v} K_{I I}^{(p)}=1\right)}{C_{2}^{(p)}}(7)
$$


According to the equation (7), the partition of viscoelastic energy release rate in each elastic spring of Kelvin Voigt chain becomes

$$
G_{v}^{(p)}={ }^{1} G_{v}^{(p)}+{ }^{2} G_{v}^{(p)}=C_{1}^{(p)} \cdot \frac{\left({ }^{u} K_{I}^{(p)}\right)}{8}+C_{2}^{(p)} \cdot \frac{\left({ }^{u} K_{I I}^{(p)}\right)}{8}
$$

${ }^{1} G_{v}^{(p)}$ and ${ }^{2} G_{v}^{(p)}$ are the energy release rate of the $\mathrm{p}^{\text {th }}$ spring in mode I and II respectively. $C_{1}^{(p)}$ and $C_{2}^{(p)}$ translate the associate viscoelastic compliances. That way, the viscoelastic energy release rate summations are given by:

$$
{ }^{1} G_{v}=\sum_{k}{ }^{1} G_{v}^{(p)} \quad \text { and } \quad{ }^{2} G_{v}=\sum_{k}{ }^{2} G_{v}^{(p)} \quad \text { with } \quad p \in\{0,1, \ldots, N\}
$$

In order to resolve the equation (7), the linear viscoelastic equations are computed according to finite difference process using the incremental constitutive equations given by [2]

$$
\Delta \varepsilon_{i j}\left(t_{n+1}\right)=\Psi_{i j k l} \cdot \Delta \sigma_{k l}\left(t_{n+1}\right)+\widetilde{\varepsilon}_{i j}\left(t_{n}\right)
$$

The terms $\Delta \varepsilon_{i j}\left(t_{n+1}\right)$ and $\Delta \sigma_{k l}\left(t_{n+1}\right)$ designate the strain and stress increments, during the time increment $\Delta t_{n} . \widetilde{\varepsilon}_{i j}(t)$ represents the global influence of the mechanical past history. $\Psi_{i j k l}$ traduces the equivalent viscoelastic compliance function.

\section{Compact Tension Shear specimen}

The Compact Tension Shear specimen, Figure 2, is used in order to operate mixed mode solicitation.

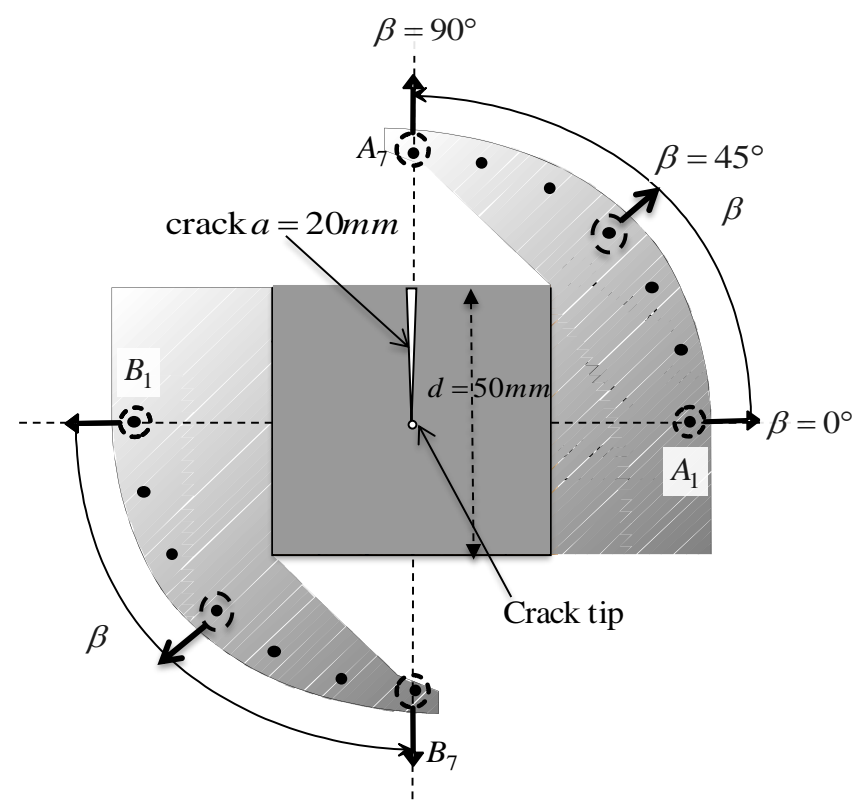

Figure 2 - Compact Tension Shear specimen [2]

This specimen is initially proposed by Richard [9] for isotropic materials and adapted to wood material by Valentin and Caumes [10]. The initial crack length chosen is $25 \mathrm{~mm}$. The external load is an unitary loading applied to a perfect rigid steel arm. Points $A_{\alpha}$ and $B_{\alpha}$ with $\alpha \in(1 \ldots 7)$ are holes where forces can be applied with the angle $\beta$ oriented according to the trigonometrically direction for different mixed mode ratios. The simple opening mode is obtained by applying opposite forces in $A_{1}$ and $B_{1}$ with $\beta=0^{\circ}$. The loading $\beta=90^{\circ}$, in $A_{7}$ and $B_{7}$, corresponds to the case of a simple shear mode. Intermediary positions induce different mixed mode configurations 


\section{Numerical algorithm}

In this section, we present the numerical procedure implemented in the finite software Castem, developed by the French Energy Atomic Commission CEA. This numerical algorithm presents relation between the uncoupling of fracture mode, viscoelastic behavior and the reliability analysis. We add in this algorithm the virtual part which computes $M \theta v$, Figure 3. We suppose that mechanical fields are known at time tn and we have fixed the time increment $\Delta t_{n}$. All properties of viscoelastic material are experimentally defined.

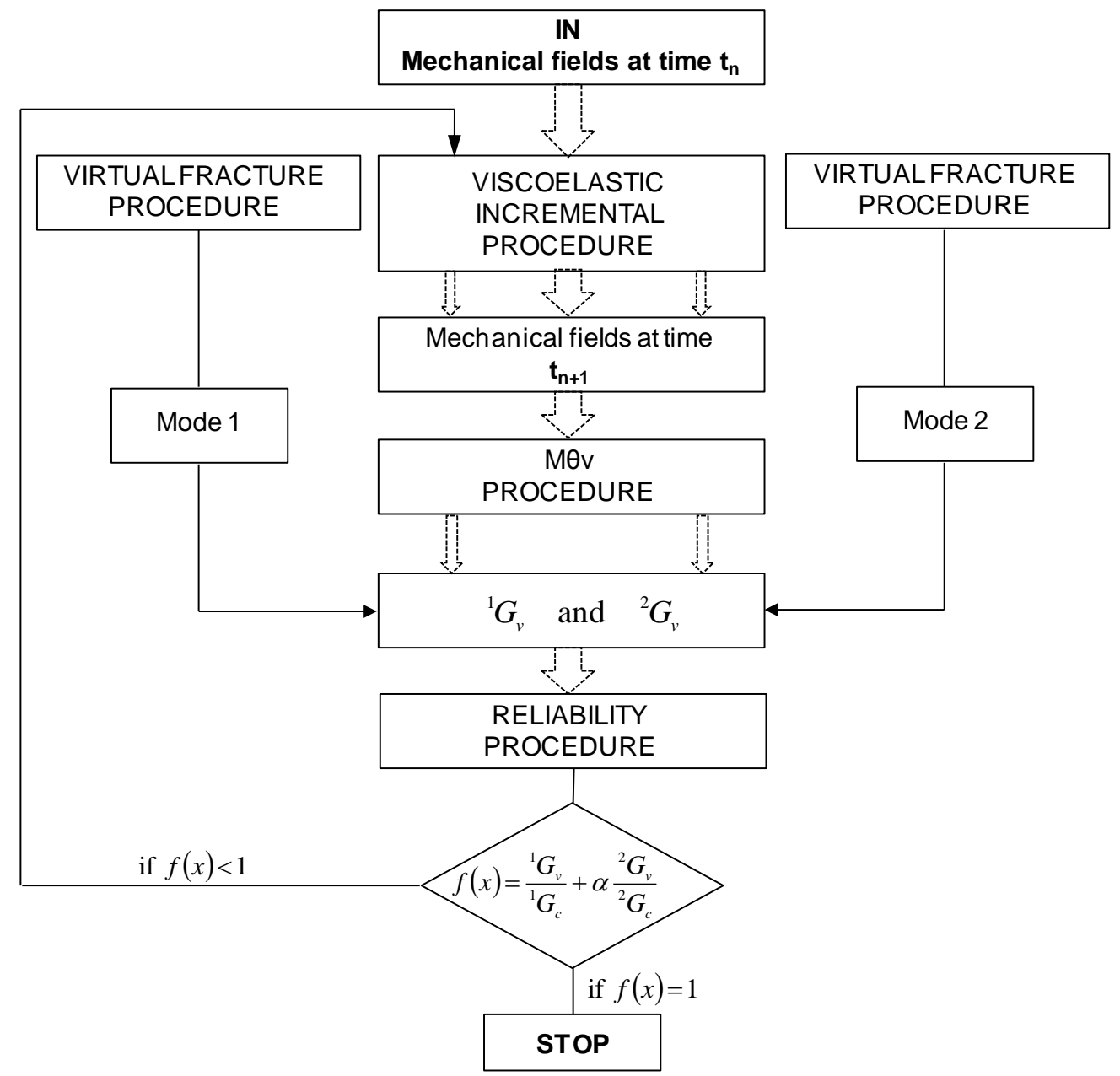

Figure 3 - Reliability and viscoelastic incremental crack algorithm

- Firstly, M $\theta$, expression (5), is computed.

- According to the viscoelastic procedure [2], the supplementary viscous load, the external load vectors are computed. Hence, obtain the nodal displacement vector incremental, and mechanical fields $\{\varepsilon\}\left(t_{n+1}\right)$, equation (10). The elastic compliance tensor $C_{1}^{(p)}$, equation (6), is introduced by using material properties. Hence, the elastic stress tensor $\left\{\sigma^{p}\right\}\left(t_{n}\right)$, and nodal load are deduced. The displacement $\left\{u^{p}\right\}$, for each Kelvin Voigt element, is computed by solving the balance equation.

- To evaluate virtual stress intensity factor ${ }^{v} K_{\alpha}^{p}$, equation (5), we use the virtual fracture procedure equation (8), is computed. Finally, the energy release rate ${ }^{\alpha} G_{v}^{p}$, and the real stress intensity factor ${ }^{u} K_{\alpha}^{p}$ and are given by $\mathrm{M} \theta$ procedure. A summation on ${ }^{\alpha} G_{v}^{p}$ gives us the energy release rate for each mode ${ }^{\alpha} G_{v}$. 
- At the end, the reliability procedure is applied in order to obtain the uncertainty parameters for the mechanical proposed model. The program is stopped if the Griffith's criteria [15] introduced by the functional $f(x)$ is verified. ${ }^{\alpha} G_{c}$ represent the critical values of the energy release rate in each mode, and $\alpha$ and unitary coefficient in our case.

\section{Numerical reliability results}

The reliability analysis is now applied to the specimen in Figure 2, where the limit state function is defined by equation (1). The random variables are described in Table 1, where the two critical energy rates are fully correlated. The applied force is defined in terms of the parameter $p$ which is equal to 1 for loading angle of $15^{\circ}$ and to 2 for loading angle of $45^{\circ}$.

\begin{tabular}{|l|c|l|c|c|}
\hline Variable & Symbol & Distribution & Mean & Standard deviation \\
\hline Longitudinal elastic modulus & $E_{x}(\mathrm{MPa})$ & lognormal & 15000 & 1500 \\
\hline Transversal elastic modulus & $E_{y}(\mathrm{MPa})$ & lognormal & 600 & 90 \\
\hline Critical energy release rate in mode 1 & ${ }^{1} G_{c}(\mathrm{~N} / \mathrm{m})$ & lognormal & 170 & 17 \\
\hline Critical energy release rate in mode 2 & ${ }^{2} G_{c}(\mathrm{~N} / \mathrm{m})$ & lognormal & 420 & 42 \\
\hline Applied force & $F(\mathrm{kN})$ & Normal & $P$ & $0.15 p$ \\
\hline Initial crack length & $a(\mathrm{~mm})$ & Deterministic & 20 & 1 \\
\hline Loading angle & $\beta\left({ }^{\circ}\right)$ & Deterministic & 45 & \\
\hline Square specimen dimension & $d$ & Deterministic & 50 & \\
\hline
\end{tabular}

Table 1 - Variables parameters

For the mixed mode angle of $15^{\circ}$, Figure 4 shows the importance of the random variables on the reliability of the structure regarding crack propagation. The material reliability is mainly controlled by the applied load, and then by the critical release energy. The failure probability is found to be $2.19 \times 10^{-2}$. The FORM procedure allows us to determine the most probable failure configuration which is defined by: $E_{x}=14688.9 \mathrm{MPa}, E_{y}=502.5 \mathrm{MPa}$, ${ }^{1} G_{c}=150.9 \mathrm{~N} / \mathrm{m},{ }^{2} G_{c}=372.8 \mathrm{~N} / \mathrm{m}$, and $F=1.19 \mathrm{kN}$. For this safety level, the partial safety factors are given by1.02, 1.19, 1.13 and 1.19 for $E_{x}, E_{y},{ }^{1} G_{c},{ }^{2} G_{c}$ and $F$, respectively.

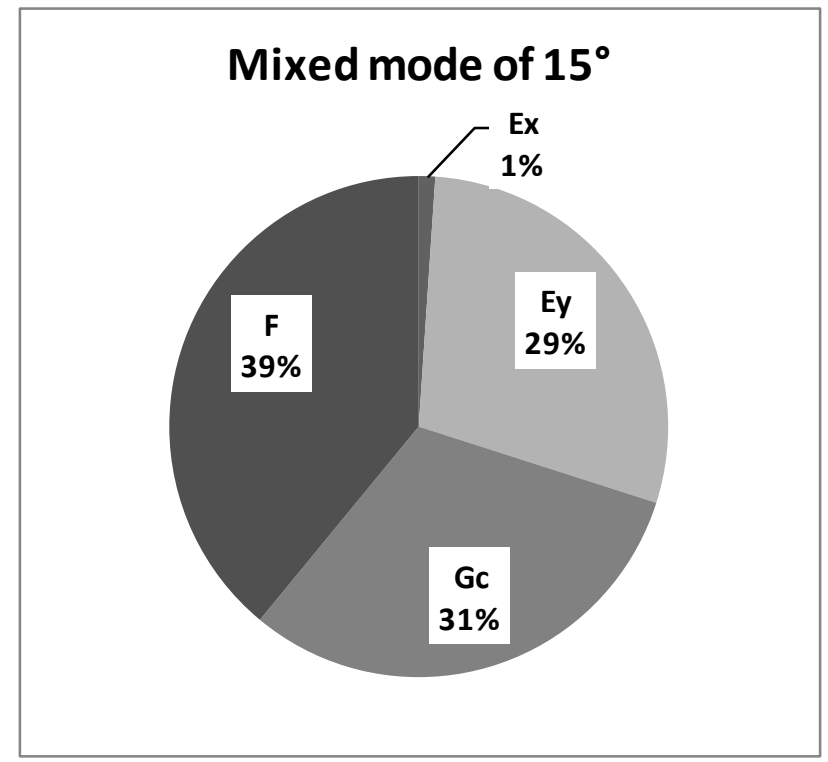

Figure 4 - Variable importance for mixed mode for $15^{\circ}$ 
In the mixed mode angle of $45^{\circ}$, Figure 5 shows the importance of the random variables on the reliability of the structure regarding crack propagation. Contrary to the case of $15^{\circ}$, the transversal elastic modulus plays the most important role in the safety of the material, with an importance of $37 \%$ instead of $29 \%$. The failure probability is found to be $1.66 \times 10^{-2}$.

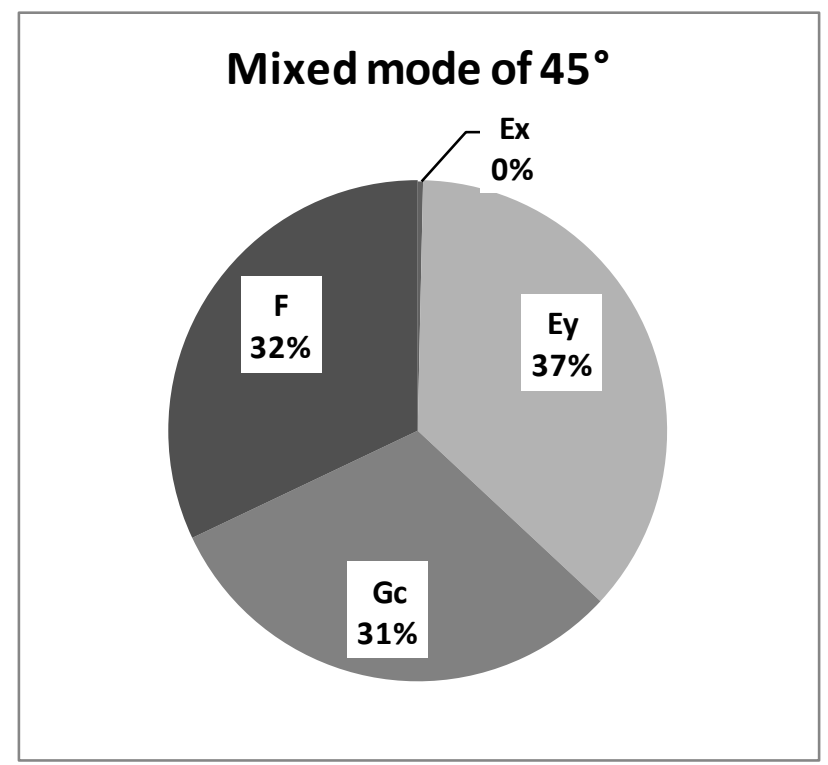

Figure 5 - Variable importance for mixed mode of $45^{\circ}$.

\section{Conclusion}

The reliability analysis coupling with mixed mode fracture and applied to viscoelastic and orthotropic materials have been studied. The different leading step in the reliability analysis consists in identifying the main set of random variables, defining the potentially critical failure modes according to a failure function and the probability of failure resolving by the First Order Reliability Method (FORM). The viscoelastic behavior has been introduced by the generalized Kelvin Voigt model solving by an incremental constitutive law. The analytical fracture separation has been introduced according to the non-dependence integral $M \theta$. In order to take into account mixed mode ratio, the CTS specimen providing the fracture during the crack tip initiation has been used. Finally, a complex subroutine combining the crack initiation, the viscoelastic and the reliability approaches has been written. The importance of the random variables on the reliability of the structure regarding crack propagation has been justified. In the numerical examples of mixed mode, it shown that the transversal elastic modulus plays the most important role in the safety of the material. In the coming work, this model will be performed in order to take into account the crack growth process.

\section{References}

[1] Jernkvist L.O., Fracture of wood under mixed mode loading II. Experimental investigation of Picea abies Lars Olof. Eng Fract Mech, 68(5), 565-576, 2001

[2] Moutou Pitti R., Dubois F., Sauvat N., Petit C., Mixed mode fracture separation in viscoelastic orthotropic media: numerical and analytical approach by the M $\theta v$-integral, Int J Fract, 125(3), 181-193, 2007.

[3] Dubois F., Chazal C., Petit C., Viscoelastic crack growth process in wood timbers: An approach by the finite element method for mode I fracture, Int J Fract, 113 (4), 367-388, 2002.

[4] Leonela E.D., Chateauneuf A., Venturinia W.S., Bressolette P. Coupled reliability and boundary element model for probabilistic fatigue life assessment in mixed mode crack propagation. Int J Fract, 32(11), 18231834, 2010.

[5] Nicholson D.W, Ni P. Extreme value probalistic theory for mixed mode britle fracture, Eng Fract Mech, 58(1), 121-132, 1997.

[6] Riahi H., Bressolette Ph., Chateauneuf A. Random fatigue crack growth in mixed mode by stochastic collocation method, Eng Fract Mech, 77(16), 3292-3309., 2010.

[7] Nother, E. Invariant variations problem, Trans Theor Stat Physics 1, 183-207, 1971. 
[8] Chen F.H.K., Shield R.T., Conservation laws in elasticity of the J-integral type, J Appl Mech Physics, 28 (1), 1-22, 1977.

[9] Richard HA., A new compact shear specimen, Int J Fract, 17(5), R105-R107, 1981.

[10] Valentin G., Caumes P., Crack propagation in mixed mode in wood: a new specimen, Wood Sci Technol, 23(1), 43-53, 1989.

[11] Ditlevsen O., Madsen H. Structural Reliability Methods. New York:John Wiley \& Sons, 1996.

[12] Lemaire M., Chateauneuf A., Mitteau J.-C., Structural reliability, John Wiley \& Sons, ISBN 978-1-84821082-0, 2009.

[13] Neves R., Chateauneuf A., Venturini S., Component and system reliability analysis of nonlinear reinforced concrete grids with multiple failure modes, Structural Safety, 30(3), 183-199, 2008.

[14] Destuynder P.H., Djaoua M., Lescure S., Some remarks on elastic fracture mechanics, J Mec Theor Appl, 2 (1), 113-135, 1983.

[15] Griffith A.A., The phenomena of rupture and flow in solids, Philos. Trans. Roy. Soc. London, 221, 163$197,1921$. 